\title{
Neuroprotective Effects of Ganoderma curtisii Polysaccharides After Kainic Acid-Seizure Induced
}

\author{
Ismael León-Rivera'*, Juana Villeda-Hernández², Elizur Montiel-Arcos ${ }^{3}$, Isaac Tello ${ }^{3}$, María Yolanda Rios', \\ Samuel Estrada-Soto ${ }^{4}$, Angélica Berenice Aguilar' ${ }^{1}$, Verónica Núñez-Urquiza' ${ }^{1}$, Jazmín Méndez-Mirón ${ }^{5}$, Victoria \\ Campos-Peña², Sergio Hidalgo-Figueroa ${ }^{6}$, Eva Hernández ${ }^{7}$, Gerardo Hurtado ${ }^{7}$
}

'Centro de Investigaciones Químicas, IICBA, Universidad Autónoma del Estado de Morelos, Avenida Universidad 1001, Col. Chamilpa 62209 Cuernavaca, Morelos, ESTADOS UNIDOS MEXICANOS.

2Instituto Nacional de Neurología

Neurocirugía Manuel Velasco Suárez. Avenida Insurgentes Sur No. 3877 Col. La Fama Tlalpan, Ciudad de México, ESTADOS UNIDOS MEXICANOS.

${ }^{3}$ Centro de Investigaciones Biológicas, Universidad Autónoma del Estado de Morelos, Avenida Universidad 1001, Col. Chamilpa 62209 Cuernavaca, Morelos, ESTADOS UNIDOS MEXICANOS

${ }^{4}$ Facultad de Farmacia, Universidad Autónoma del Estado de Morelos, Avenida Universidad

1001, Col. Chamilpa 62209 Cuernavaca

Morelos, ESTADOS UNIDOS MEXICANOS.

${ }^{5}$ Facultad de Ciencias Biológicas, Universidad Autónoma del Estado de Morelos, Avenida

Universidad 1001, Col. Chamilpa 62209 Cuernavaca, Morelos, ESTADOS UNIDOS MEXICANOS.

${ }^{6}$ CONACYT-IPICYT Consorcio de Investigación, Innovación y Desarrollo para las Zonas Áridas, 78216 San Luis Potosí, ESTADOS UNIDOS MEXICANOS.

7 Instituto Nacional de Salud Pública. Avenida Universidad 565, Col. Santa María Ahuacatitla Cuernavaca, Morelos, ESTADOS UNIDOS MEXICANOS.

\section{Correspondence}

\section{Ismael León-Rivera}

Centro de Investigaciones Químicas, IICBA, Universidad Autónoma del Estado de Morelos, Avenida Universidad 1001

Col. Chamilpa 62209 Cuernavaca, Morelos, ESTADOS UNIDOS MEXICANOS.

Phone no: 7773297997

E-mail: ismaelr@uaem.mx

History

- Submission Date: 04-07-2019;

- Review completed: 18-07-2019;

- Accepted Date: 31-07-2019.

DOI : 10.5530/pj.2019.11.164

Article Available online

http://www.phcogj.com/v11/i5

\section{Copyright}

(C) 2019 Phcogj.Com. This is an openaccess article distributed under the terms of the Creative Commons Attribution 4.0 International license.

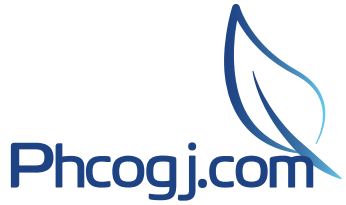

\section{ABSTRACT}

Background: Epilepsy is one of the major neurological disorders affecting world population Although, some Ganoderma species have shown neuroprotective activities, the effects of polysaccharides isolated from Ganoderma curtisii on epileptic seizures have not been reported. Objective: The aims of the present study were to determine whether treatment with a polysaccharide fraction (GCPS-2) from a Mexican Ganoderma curtisii strain can reduce seizures, and the increases in the levels of apoptotic molecules and inflammatory cytokines in kainic acid-induced seizure mouse model. Materials and Methods: Rats were separated in groups: Control group received 2.5\% Tween 20 solution; GCPS-2 groups were administered GCPS-2 $(10,40$, or $80 \mathrm{mg} / \mathrm{kg})$; KA group received KA $10 \mathrm{mg} / \mathrm{kg}$; GCPS-2+KA received GCPS2 and 30 min later KA. Pathological changes in neuronal morphology, expression of B-cell lymphoma-2, and pro-inflammatory cytokines (interleukin1- $\beta$ and tumor necrosis factor- $\alpha$ ) in the rat hippocampus and cortex were determined by immunohistochemistry. Results: Ganoderma curtisii soluble polysaccharides (GCPS-2) inhibited convulsions in rats. Moreover, treatment with GCPS-2 reduced the increased levels of apoptotic signaling molecules (Bcl-2) and proinflammatory mediators (in the kainic acid-treated hippocampus and cortex). Conclusion: Ganoderma curtisii soluble polysaccharides have a neuroprotective potential against epilepsy, partially through its ability to inhibit neurotoxic events in the in vivo hippocampus and cortex. Key words: Ganoderma curtisii, $\beta$-glucan, Neuroprotective, Anticonvulsant, Anti-Inflammatory.

\section{INTRODUCTION}

Epilepsy is one of the major neurological disorders affecting approximately $1 \%$ of world population. Currently available antiepileptic drugs (AED): are associated with severe side-effects, does not affect the underlying disorder, and about a third of patients are refractory to pharmacotherapies. A series of biophysiological and pathological reactions following seizures contribute to subsequent neuronal cell death. Apoptosis has been found as a way of cell death in pathological responses postseizures. Determining the underlying pathological mechanisms and developing potential therapeutic strategies for epileptic seizures are important research areas. The development of effective AED has been unfavored by the limited knowledge of the pathogenesis of this neurological disorder.

Kainic acid was one of the first compounds used to study temporal lobe epilepsy (TLE). The administration of this L-glutamate analog causes neuronal depolarization and seizures, preferentially targeting the hippocampus. Injected rodents with kainic acid show recurrent seizures of variable frequency and hippocampus-restricted injuries. Neurodegeneration caused by injection of KA has been widely used in experiments to investigate mechanisms of excitotoxicity and possible pharmacological intervention against the seizure events. KA preferentially damages the pyramidal neurons of the CA3 area of the hippocampus, surviving neurons in the CA1 area of the hippocampus became hyperexcitable. ${ }^{2}$

Seizures are associated with some events such as inflammation, oxidative stress, neurotransmitter release, astrocyte proliferation, and microglia activation. Neuroinflammation plays a critical role in the pathogenesis of some neurodegenerative diseases. Although microglial activation is beneficial because it tends to diminish further injury and contributes to the repair of damaged tissues. Chronic activation of microglial cells endangers neuronal survival through the release of various proinflammatory molecules and neurotoxins such as nitric oxide (NO), proinflammatory cytokines such as tumor necrosis factor alpha (TNF- $\alpha$ ). Therefore, attenuation of microglial activation is considered a valuable strategy for the prevention and treatment of neurodegenerative diseases. ${ }^{3}$

Polysaccharides isolated from Ganoderma lucidum have been reported as neuroprotective agents against ischemia, inflammation, and neuroapoptosis. ${ }^{4}$ Ganoderma curtisii is a mushroom with few reported scientific studies, but there is not reports about the isolation of polysaccharides from $G$. curtisii nor the biological activity of these polysaccharides.

Therefore, this study aimed to assess the anticonvulsant and neuroprotective effect of

Cite this article: León-Rivera I, Villeda-Hernández J, Montiel-Arcos E, Tello I, Rios MY, EstradaSoto S, et al. Neuroprotective Effects of Ganoderma curtisii Polysaccharides After Kainic AcidSeizure Induced. Pharmacog J. 2019;11(5):1046-54. 
Ganoderma curtisii polysaccharides in rats. Seizures were induced by intraperitoneal injection of kainic acid-seizure. Furthermore, whether the neuroprotective effects of GCPS-2 are associated with neuronal apoptosis and/or anti-inflammatory effects was investigated.

\section{MATERIALS AND METHODS}

\section{Ganoderma curtisii strain}

Ganoderma curtisii fruiting bodies were collected from a forest in the state of Morelos, Estados Unidos Mexicanos in August 2010. Identification of the mushroom was performed by M. C. Elizur Montiel and Dr. Isaac Tello, Laboratorio de Micología, Centro de Investigaciones Biológicas (CIB), Universidad Autónoma del Estado de Morelos (UAEM), Estados Unidos Mexicanos, and a voucher of the specimen (COBIOCh-UAEMOR-CF-G01) is deposited in the CIB Herbarium, UAEM.

Ganoderma curtisii mycelium strain was isolated from the fruiting bodies. The strain of G. curtisii was cultivated for 7 days on PDA slants agar at $24^{\circ} \mathrm{C}$, and it was re-inoculated every 4 weeks. The mycelium (inoculum, 2\% wet weight) was transferred to a $250 \mathrm{~mL}$ Erlenmeyer flask containing $100 \mathrm{~mL}$ of liquid medium $\left(\right.$ at $24^{\circ} \mathrm{C}, \mathrm{pH}=6.0$ ). The mycelium was incubated, on a rotary shaker $(200 \mathrm{rpm})$ for 10 days.

In the submerged cultivation, mycelium was separated from the cultivation broth by vacuum filtration. The insoluble polysaccharides were separated by addition of two volumes of ethanol to the culture medium, and then stored at $4{ }^{\circ} \mathrm{C}$ overnight. The precipitated and culture medium was centrifuged and filtered. The culture medium was liofilized yielding a dark brownish colored semi-solid mass (GCPS-2).

\section{Phytochemical screening of GCPS-2}

Preliminary phytochemical screening of GCPS-2 was performed for the detection of chemical constituents: alkaloids, steroids, terpenoids, flavonoids, tannins, phenolics, saponins, carbohydrates, proteins and amino acids. ${ }^{5}$

After the positive result for carbohydrates, the sugar composition of GCPS-2 (40 mg) was determined by acidic hydrolysis with $0.5 \mathrm{M}$ trifluoroacetic acid for $5 \mathrm{~h}$. After this time, the aqueous phase from the acid hydrolysis reaction was neutralized with $\mathrm{Na}_{2} \mathrm{CO}_{3}$ solution and lyophilized to give a white solid. Furthermore, the residue was dissolved in $\mathrm{CH}_{3} \mathrm{CN}$ and analyzed by the HPLC system fitted with a $\mathrm{NH}_{2}$ column (Supelco, $250 \mathrm{~mm} \times 10 \mathrm{~mm} ; 10 \mu \mathrm{m}$ ), an isocratic elution of $\mathrm{CH}_{3} \mathrm{CN}: \mathrm{H}_{2} \mathrm{O}$ $(85: 15 \mathrm{v} / \mathrm{v})$, at a flow rate of $1 \mathrm{~mL} / \mathrm{min}$, and a sample injection of 100 $\mu \mathrm{L}(4 \mathrm{mg} / \mathrm{mL})$ of standard carbohydrate solutions. Identification of the sugar components was performed by coelution experiments with standard samples (galactose, glucose). The HPLC system consisted of an Agilent HPLC system, fitted a 1100 series pump, Rheodyne injector, and a 1100 variable wavelength UV-Vis detector at $210 \mathrm{~nm}$.

A sample of GCPS-2 $(20 \mathrm{mg})$ was dissolved in $\mathrm{D}_{2} \mathrm{O}(0.6 \mathrm{~mL})$ and then analyzed by NMR using an Agilent 400 NMR spectrometer. One- $\left({ }^{1} \mathrm{H}\right.$, ${ }^{13} \mathrm{C}$ ) and two-dimensional NMR (COSY and HETCOR) experiments were obtained with a $5-\mathrm{mm}$ probe at $25^{\circ} \mathrm{C}$. Proton chemical shifts were referenced to the residual signal for HDO. Positive-ion HRMALDITOF MS data of GCPS-2 was acquired using a Shimadzu LCMS 8030 spectrometer, equipped with a laser of nitrogen at $337 \mathrm{~nm}$. The analyte was concentrated using Zip Tip $\mathrm{C}_{18}$ (Millipore), eluted with a solution $\left(80 \% \mathrm{v} / \mathrm{v} \mathrm{CH}_{3} \mathrm{CN} / 0.5 \%\right.$ trifluoroacetic acid) saturated with a-cyano4-hydroxycinnamic acid (Sigma), applied on the metallic sample plate, dried, and analyzed in reflector mode.

\section{Animals and Kainic acid-induced seizure model}

Male Wistar rats were purchased from Harlan (Mexico) weighting 250 - $300 \mathrm{~g}$, with 7 - 8 weeks old. Rats were housed in the animal house of the Laboratorio de Enfermedades Neurodegenerativas, Instituto Nacional de Neurología y Neurocirugía. The animals were housed randomized in groups of 5 per cage, fed with normal commercial pellet diet (Harlan, Mexico), free access to water, and maintained under laboratory conditions (temperature $24-25^{\circ} \mathrm{C}$, under a $12 \mathrm{~h}$ light-dark cycle) at least 3 weeks to adapt to the laboratory environment prior to experiments. All animal procedures were performed according with the Mexican Guidelines for Care and Use of Laboratory Animals (Norma Oficial Mexicana NOM-062-ZOO-1999) and the Guidelines for the Care and Use of Mammals in Neuroscience and Behavioral Research from the Society of Neuroscience. The minimum number of animals $(n=5$ for each group) and duration of observation required to obtain consistent data were employed. Seizures were induced by intraperitoneal (ip.) injection of KA (10 mg/kg animal). Rats injected with $2.5 \%$ Tween 20 solution alone were used as control group.

\section{Drugs administration}

In order to investigate the effects of GCPS-2 in preventing epileptic seizures, the drug was freshly prepared in $2.5 \%$ Tween 20 solution. Experiments were performed as following: GCPS-2 (10, 40, or $80 \mathrm{mg} /$ $\mathrm{kg}$ ) or kainic acid (KA, $10 \mathrm{mg} / \mathrm{kg}$ ) were dissolved in $2.5 \%$ Tween 20 solution; and GCPS-2 or KA were administered i.p. to animals. Rats were separated in groups ( 5 rats per group): Control group received 2.5\% Tween 20 solution; GCPS-2 groups were administered GCPS-2 (10, 40, or $80 \mathrm{mg} / \mathrm{kg})$; KA group received $\mathrm{KA} 10 \mathrm{mg} / \mathrm{kg}$; GCPS-2+KA received GCPS-2 and 30 min later KA. ${ }^{6}$

\section{Monitoring of KA-induced seizures}

Animals of each group after KA treatment, were placed separately into transparent plexiglas cages $(25 \times 15 \times 10 \mathrm{~cm})$ and monitored for up $60 \mathrm{~min}$ in order to evaluate the time of seizure onset. The severity of seizures was classified into five stages: stage 1 , characterized by facial movements; stage 2 , characterized by head nodding and myoclonic twitching; stage 3 , characterized by forelimb clonus with lordotic posture; stage 4 , characterized by forelimb clonic seizures with reared posture, stage 5 , characterized by tonic-clonic seizures without postural control; and stage 6 , characterized by death of rats. In the present study, rats exhibiting seizures with at least stage 3 severity were considered positive for seizure onset.

\section{Nissl staining of rat hippocampus and cortex}

Brain sections from sacrificed animals were exposed to the Nissl stain for the assessment of neuronal cell loss at hippocampus and cortex, as previously described. Two days after KA treatment animals were deeply anesthetized with pentobarbital $(40 \mathrm{mg} / \mathrm{kg}$, intraperitoneally) and then transcardially perfused with cold saline followed by $4 \%$ paraformaldehyde in PBS $(0.1 \mathrm{~mol} / \mathrm{L} ; \mathrm{pH}$ 7.4). Brains were removed, washed in PBS, cryoprotected with $30 \%$ sucrose in PBS, and frozen in powdered dry ice. Coronal sections $(6 \mu \mathrm{m})$ were cut at the level of the dorsal hippocampus (820 HistoSTAT microtome). Every fourth sections were mounted on slides, dehydrated and rehydrated in graded ethanols and xylenes, respectively and then incubated in $1 \%$ cresyl violet for 30 seconds. Sections were then decolorized in acetic acid dehydrated, and coverslipped. Sections were observed with a binocular microscope (Primo Star, Carl Zeiss). ${ }^{7}$

\section{Immunohistochemistry staining}

Brain sections were prepared for immunohistochemistry staining following a procedure optimized in our laboratory. In brief, after two consecutive PBS washes, sections were incubated overnight at $4^{\circ} \mathrm{C}$ in PBS-BSA containing the primary antibodies: B-cell lymphoma-2 (Bcl-2, 1:50); glial fibrillary acidic protein (GFAP, 1:100 dilution), IL$1 \beta$ (1:100 dilution), mouse anti-Hsp60 monoclonal antibody (1:1000 
dilution), mouse anti-Hsp70 monoclonal primary antibody (1:1000 dilution), and mouse anti-neuronal nuclei (NeuN, 1:200 dilution). The sections were subsequently washed and incubated with biotinconjugated secondary antibodies, followed by an avidin biotin complex kit. The stained sections were examined under a microscope (Primo Star, Carl Zeiss).

\section{Counting of hippocampal CA1, CA2, and CA3 neurons}

The number of hippocampal CA1, CA2, or CA3 neurons was quantified using alternate sections prepared from the coronal brain slices of animals. Neurons with visible nuclei were counted using a light microscope according a procedure optimized in our laboratory (Primo Star, Carl Zeiss).

\section{Statistical analysis}

Statistically comparisons for significance among the different groups of animals were performed by ANOVA and Tukey test. Statistical comparisons for significance between cells were performed via Student's $\mathrm{t}$-test. The differences were considered statistically significant when $\mathrm{P}<$ 0.05 . Data are expressed as the arithmetic mean \pm standard error.

\section{RESULTS}

\section{Phytochemical screening of GCPS-2}

The chemical tests used for preliminary phytochemical analysis indicated that no triterpene, flavonoid, saponin or protein compounds were detected in this fraction. The only positive test was for saccharides. The yield of the soluble polysaccharide fraction GCPS- 2 was $3 \mathrm{~g} / \mathrm{L}$ Sugar compositional analysis of GCPS-2 was performed by acid hydrolysis of the polysaccharide and HPLC analysis of the hydrolyzed, indicated that polysaccharide was composed by glucose $\left(t_{R}=12.2 \mathrm{~min}\right)$ units.

In the ${ }^{1} \mathrm{H}$ NMR spectrum of GCPS-2 (Figure 1), a weak anomeric signal was identified at $5.18 \mathrm{ppm}$ with a coupling constant $\left({ }^{3} \mathrm{~J}_{\mathrm{H} 1-\mathrm{H} 2}\right)$ of 3.1 Hz. Additionally, another weak anomeric signal was observed at $5.22 \mathrm{ppm}$ with a similar coupling constant value. These values of ${ }^{3} \mathrm{~J}_{\mathrm{H} 1-\mathrm{H} 2}$ indicate an alpha $(\alpha)$ configuration for glucose units. There were also two higher intense anomeric signals at 4.43 and $4.62 \mathrm{ppm}$ with a ${ }^{3} \mathrm{H}_{\mathrm{H} 1 \mathrm{H} 2}$ $=9.1 \mathrm{~Hz}$ corresponding to a beta ( $\beta$ configuration. The proportions of the integrated peak areas for these signals were 1:1:9:9 respectively, indicating that $\beta$-glucan was the most abundant compound in GCPS- 2 . The ${ }^{13} \mathrm{C}$ NMR spectrum of GCPS-2 (Figure 2) show signals at 98.2 and $95.9 \mathrm{ppm}$ corresponding to a $\beta$ configuration, and signal at $94.5 \mathrm{ppm}$ corresponding to an a configuration. ${ }^{8}$ The branching points at C-6 were shown by the $-\mathrm{CH}_{2}-\mathrm{O}$ - signals at $66.7 \mathrm{ppm}$ in the spectrum, compared to the unsubstituted $-\mathrm{CH}_{2}-\mathrm{OH}$ signals at $63 \mathrm{ppm} .{ }^{9}$ In addition, MALDITOF mass spectrometry indicated signals in the range 40000 $45000 \mathrm{Da}$ for GCPS-2.

\section{GCPS-2 pretreatment inhibits seizures}

The KA excitotoxicity was stablished to induce seizures; behavioral changes and neuro-histochemical features similar to those found in in patients with TLE. Rats administered with $\mathrm{KA}(10 \mathrm{mg} / \mathrm{Kg})$ showed the stages 1 to 5 of seizure severity including the stage 6 (death of one animal). The first stage of neurotoxicity in rats occurred at $90 \mathrm{~min}$ after KA administration. To determine the effects of GCPS- 2 on KA-induced seizures, various doses of GCPS-2 $(10,20,40,80 \mathrm{mg} / \mathrm{Kg})$ were i.p. administered 30 minutes before the KA injection. The effect of GCPS2 in inhibiting seizures was not dose-dependent, at all doses rats only showed behavior of stage 1 . Rats treated with GCPS- 2 at various doses $(10,20,40,80 \mathrm{mg} / \mathrm{kg})$ did not develop any stage of seizure severity.

\section{Neuroprotective effects of GCPS-2}

Hippocampal and cortex sections of rats from all groups were stained with hematoxylin and eosin $(\mathrm{H} \& \mathrm{E})$ to detect degenerated neurons (Figure 3). We can observe that in control group there was no evidence of neuronal damage. A preservation of cortex neurons was found in rats treated with GCPS-2 $(10 \mathrm{mg} / \mathrm{kg})$. The majority of neurons in cortex sections in the group treated with $\mathrm{KA}(10 \mathrm{mg} / \mathrm{Kg})$, appeared shrunken with eosinophilic cytoplasm, triangulated pyknotic nuclei, and edema (Figure 3C). In contrast, rats pre-treated with GCPS-2 (10 $\mathrm{mg} / \mathrm{Kg}$ ) did not show a significant decrease in the number of neurons after KA injection (Figure 3D). In the graph (E), we can observe that there are significant differences $(p=0.0058)$ in cell damage, between

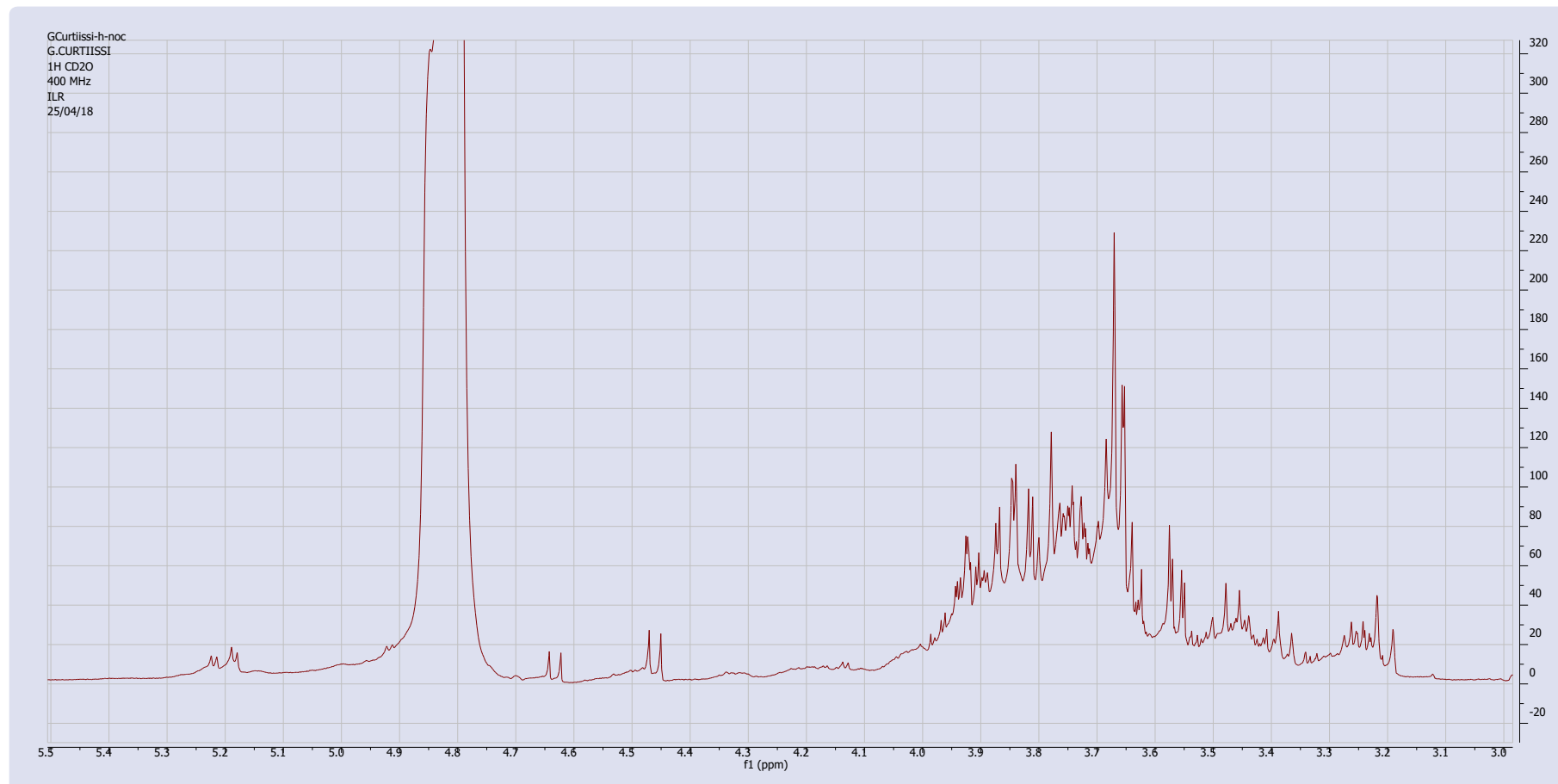

Figure 1: 'H NMR spectrum of GCPS-2 (400 MHz, $\left.\mathrm{D}_{2} \mathrm{O}\right)$. 


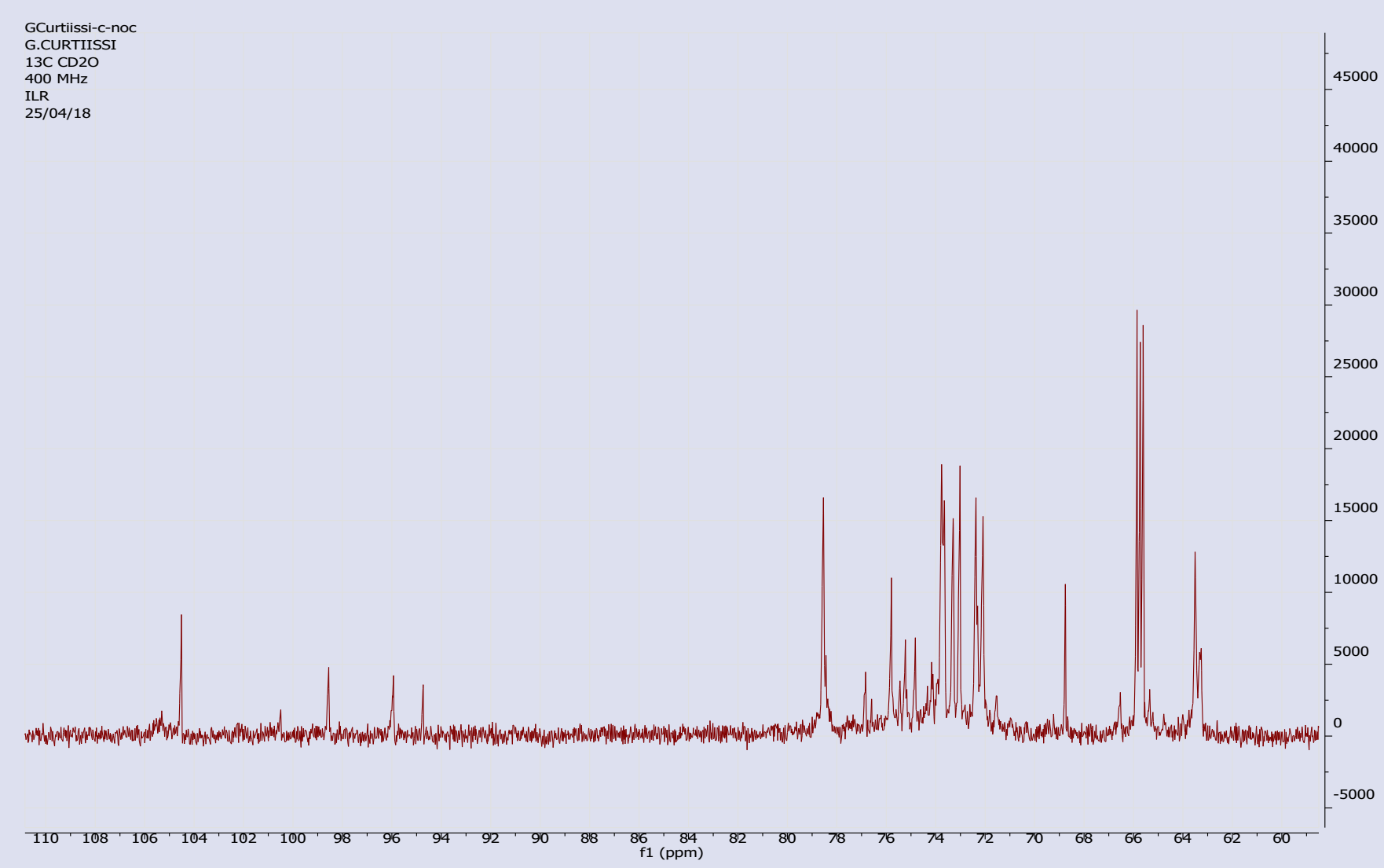

Figure 2: 13C NMR spectrum of GCPS-2 (400 MHz, D2O).

different treatments. Brain sections were also analyzed to determine the effect of these treatments on astrocytes activation. Rats treated with GCPS-2 $(10 \mathrm{mg} / \mathrm{Kg})$ displayed a staining for GFAP very similar to that of the control group, and astrocytes showing few morphological changes. Reactive astrocytes with increased GFAP labeling and altered morphology (thickening of their processes) were observed in animals that received KA injection. Numerous GFAP-positive astrocytes were present in hippocampus and cortex sections of rats treated with KA. Finally, animals pre-treated with GCPS-2 $(10 \mathrm{mg} / \mathrm{Kg})$ before the insult with KA displayed a weak staining for GFAP, and few activated astrocytes, similar to control group (Figure 4). Pretreatment with GCPS-2 inhibited the astrocyte activation.

\section{Effect of GCPS-2 on HSP70 and HSP60 expression}

In order to determine whether GCPS- 2 protects against kainic acid neurotoxicity the HSP70 or HSP60 expression levels in the cortex of different experimental rat groups were determinated. As shown in Figure 5, HSP70 expression levels were significantly higher in the KA group compared with the control group. Administration of GCPS-2 (B) did not induce an increase in the expression of HSP70, being the levels similar to that of control group (A). Kainic acid induced an elevated expression of HSP70, meanwhile in group with pretreatment of GCPS2, the HSP70 immunoreactivity decreased in comparison with KA group as shown in (D). The expression of HSP60 was determined also by immunostaining (Figure 6). In KA group, HSP60 immunoreactivity was increased in the hippocampus and cortex regions (C). In animals pretreated with GCPS-2 and a posterior KA administration, HSP60 immunoreactivity decreased in comparison with KA group (D) (Figure 6). Animals treated with GCPS-2 (B), showed an expression of HSP60 similar to that of control group (A).

\section{Effect of GCPS-2 on NeuN}

NeuN labeling was used to encompass neuronal cell losses by staining for detecting neurons in the process of degeneration. After KA injection, the numbers of NeuN-positive cells in cortex sections were significantly decreased. Also, a significative loss of cells, interstitial edema and cellular damage was observed in the cortex. Group pre-treated with GCPS-2 showed a similar expression of NeuN-positive neurons to control group (Figure 7). Group that received GCPS-2, showed some cells in the process of neurodegeneration (B).

\section{GCPS-2 attenuates KA-induced apoptotic molecules expression}

To examine whether GCPS-2 pretreatment inhibits KA-induced apoptosis, the expression levels of $\mathrm{Bcl}-2$ an apoptotic molecule was determined in samples of hippocampus and cortex brain tissues. Basal expression of $\mathrm{Bcl}-2$ was detected in hippocampus and cortex samples from the control group. Meanwhile, animals treated with GCPS-2 showed a level of Bcl-2 expression similar to that of control group. A great $\mathrm{Bcl}-2$ positive staining was visible in hippocampus and cortex sections of rats subjected to KA when compared with control group, demonstrating that KA treatment significantly enhanced the expression of Bcl-2. Furthermore, the increased levels of Bcl-2 in the KA-treated hippocampus and cortex sections was significantly attenuated by pretreated with GCPS-2, compared with KA group (Figure 5).

\section{GCPS-2 downregulates pro-inflammatory mediators in} KA-treated rats

To determine whether GCPS- 2 affects the expression of inflammatory mediators such as IL- $1 \beta$ and TNF- $\alpha$, which may be produced by 


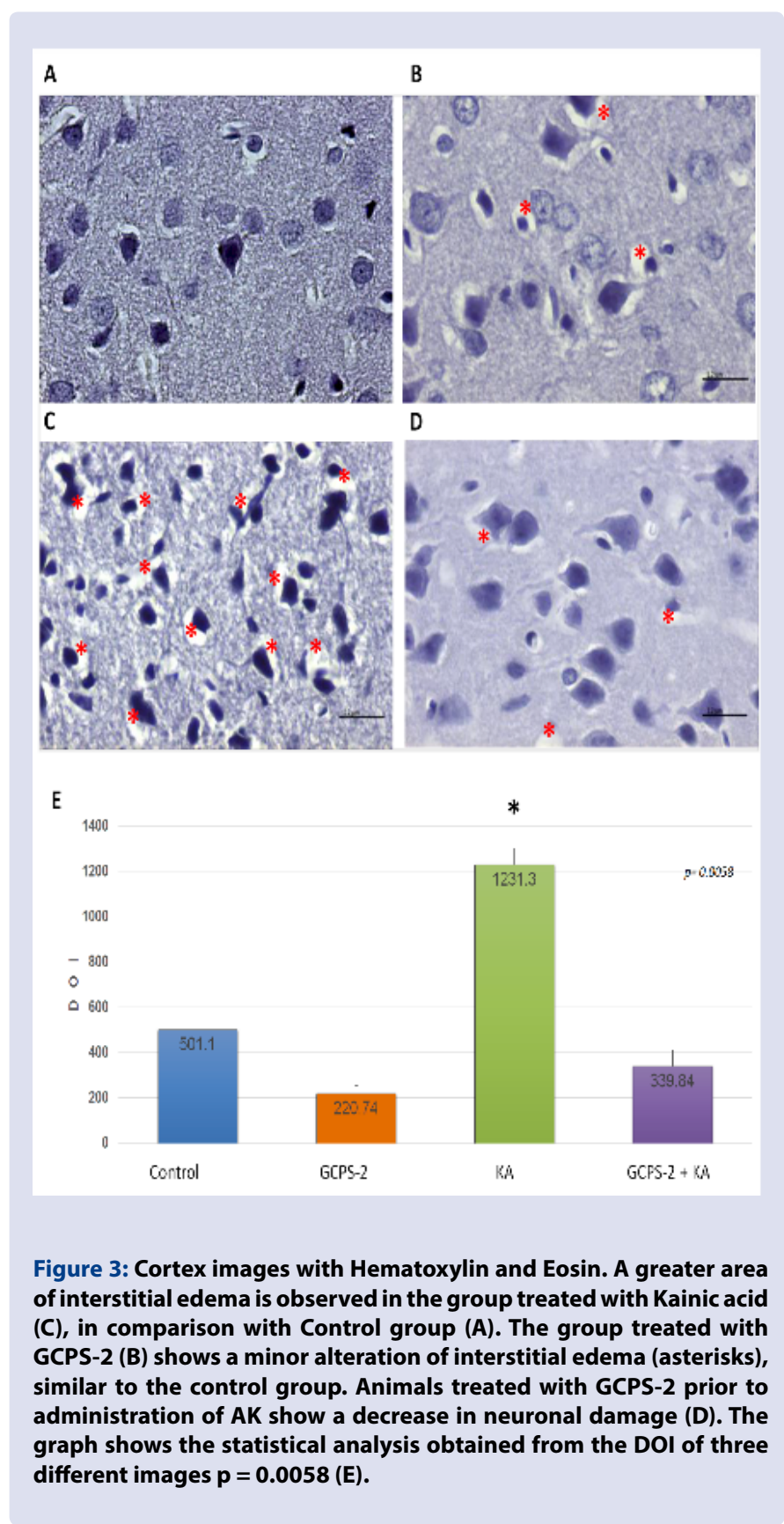

activated microglia, in the hippocampus and cortex sections were stained. The results obtained (Figure 6) demonstrate that KA treatment resulted in microglial activation and an increase in IL- $1 \beta$ and TNF- $\alpha$ in hippocampus and cortex. GCPS- 2 pretreatment inhibited these increases in inflammatory mediators. These results suggest that GCPS-2 protects hippocampal and cortex neurons by inhibiting the production of pro-inflammatory mediators.

\section{DISCUSSION/CONCLUSION}

Studies on Ganoderma species have been performed mainly on Asian and European strains, but few studies have considered Mexican strains. And besides, Ganoderma curtisii is a mushroom that has been few studied. This is the first report of isolation of polysaccharides from Ganoderma curtisii. Aguilar et al. reported the polyphenol content, and Yang et al., isolated lanostane triterpenoids from fruiting bodies of $G$. curtisii, but they did not isolate polysaccharides. ${ }^{10,11}$

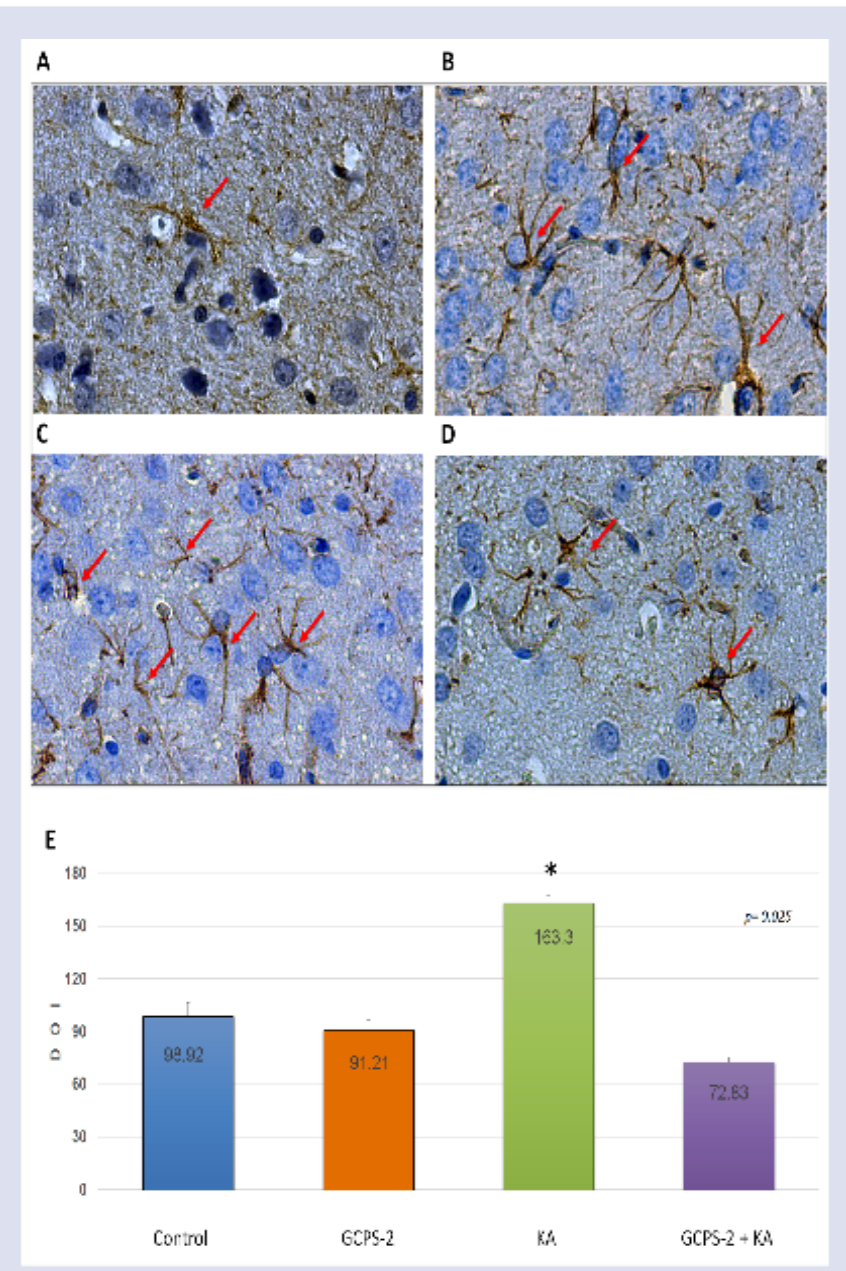

Figure 4: Photomicrographs of astrocytic activity observed in the cortex brain from different treatments: A) Control group showing normal neurons and GFAP level; B) GCPS-2 group showing normal neurons and GFAP level similar to Control group after administration of GCPS-2 (10 mg/Kg); C) kainic acid group showing abundant neuronal loss, besides a high induction of GFAP after injection of kainic acid (10 mg/kg); D) GCPS-2 + KA group with GCPS-2 (10 mg/ $\mathrm{kg})$ pretreatment and later administration of kainic acid $(10 \mathrm{mg} / \mathrm{kg})$ showing less degenerate neurons and lower GFAP level than kainic acid group; E) Higher activity is observed in the animals treated with Kainic acid, while the treatment with Ganoderma curtisii and the treatment of GCPS-2 + KA have similar damage to Control group.

Several reports have shown that $\beta$-glucans have been isolated from fruiting bodies of mushrooms, but few reports mention the isolation of $\beta$-glucan from submerged culture of mycelium. ${ }^{12,13}$ This is the first report of $\beta$-glucan isolation from submerged cultivation of $G$. curtisii.

Although new antiepileptic drugs have shown advantages as anticonvulsants, their efficiency is limited. The search for new molecules with neuroprotective activities has been performed on plants and mushrooms. There are several mushrooms which have been reported as an interesting source of metabolites, with the ability to promote cure on brain affections. Polysaccharides are one type of metabolites that have shown neuroprotective properties against brain injury induced by neurotoxins and stroke. The beneficial neuroprotective effects of polysaccharides consider the capacity to suppress neuroinflammation and excitotoxicity with minimal side effects. There are few reports 


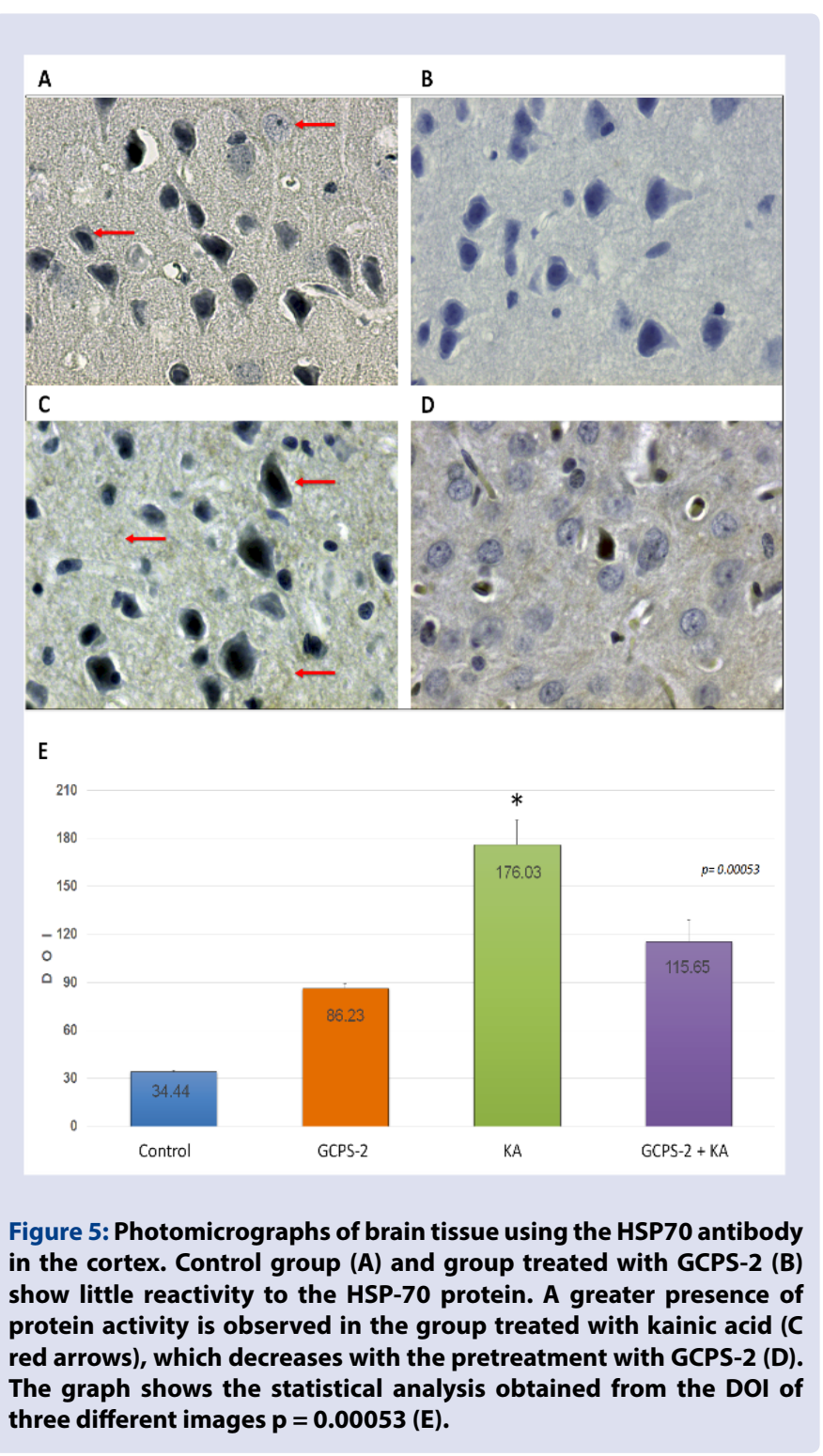

describing anticonvulsant activity of polysaccharides isolated from Ganoderma species. ${ }^{14,15}$ The anticonvulsant activity induced by GCPS- 2 is similar to that reported by us with an oligosaccharide fraction from a Ganoderma lucidum strain collected in a Mexican forest. ${ }^{16}$ These results suggest that GCPS-2 is a natural compound with potential anticonvulsant activity.

Kainic acid administered to rats induced seizures and caused hippocampal and cortex neuronal death. The neuronal death may be due to apoptosis triggered by the activation of several transduction factors as caspase- $3, \mathrm{Bcl}-2$, and $\mathrm{Bax},{ }^{17}$ suggesting that controlling apoptosis may be an important protection of hippocampus and cortex following epileptic seizures. Pretreatment with GCPS-2 protected the hippocampus and cortex neurons against KA-induced excitotoxicity. And besides, GCPS-2 prevented the increase of $\mathrm{Bcl}-2$ levels following the KA injection. These results suggest that GCPS-2 pretreatment attenuated the apoptotic signal induced by KA treatment, resulting in protection from neuronal degeneration. GCPS-2 pretreatment also protected the hippocampal and cortex neurons against neurotoxicity.

As a default response to injuries caused by excitotoxicity and a variety of neurodegenerative diseases (including epilepsy), cells induce a variety of proteins among them heat shock proteins that confers cellular

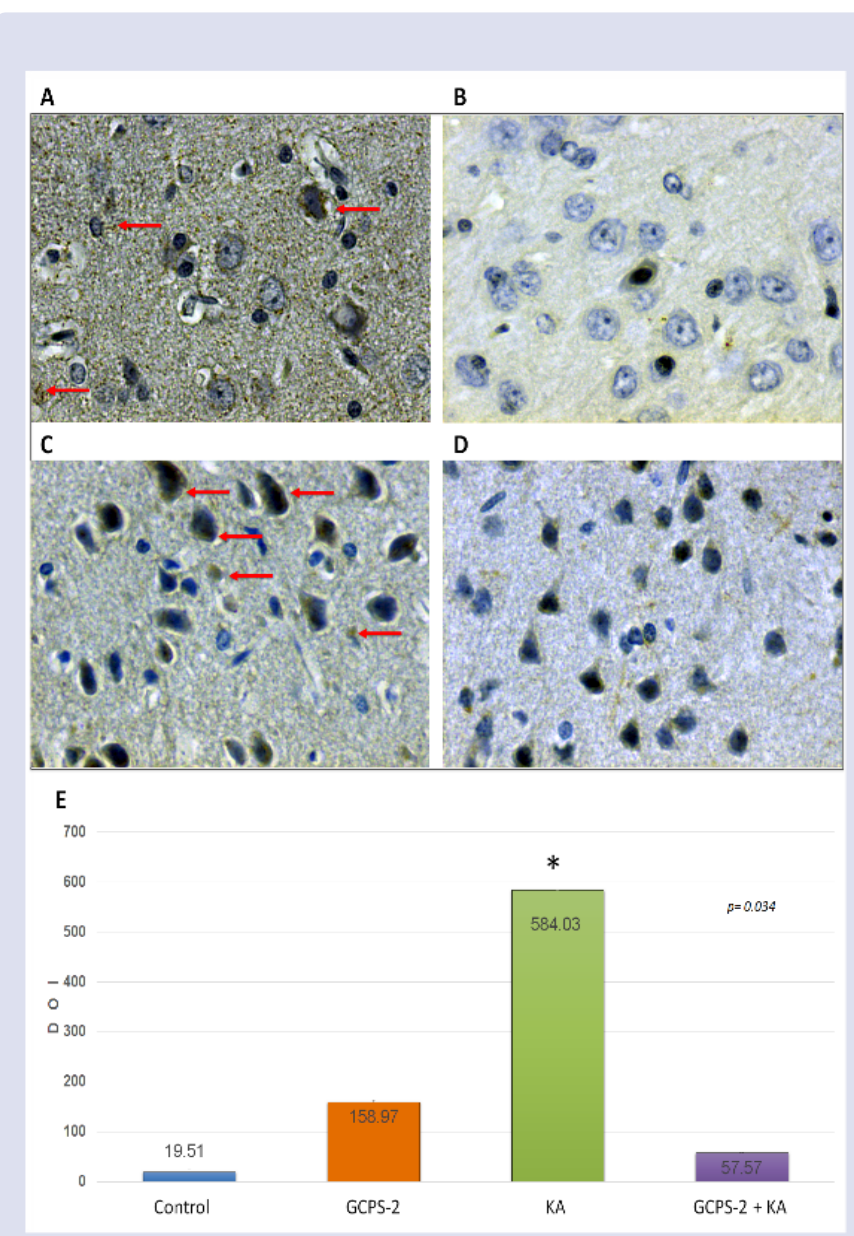

Figure 6: Photomicrographs of cortex brain tissue sections: A) control group showing normal neurons and HSP60 level; B) kainic acid group showing abundant neuronal loss, besides a high induction of HSP60 after injection of kainic acid; C) GCPS-2 group, showing normal neurons and Hsp60 level similar to control group; D) GCPS-2 + KA group with GCPS-2 pretreatment and later kainic acid showing less degenerate neurons and lower HSP60 level than kainic acid group.

protection against insults. ${ }^{18}$ In the present study, the expression levels of Hsp60 or Hsp70 expression increased by the administration of KA. These results and the Bcl-2 expression suggest that neuronal apoptosis could be implicated in the kainic acid-seizure induction. KA may initiate a neuronal apoptosis cascade that contributes to substantial neuronal damage, convulsions and behavioral impairment. The pretreatment with GCPS- 2 reduced the expression of HSP60 or HSP70 after the administration of KA, showing the neuroprotective effects of GCPS-2. The results of the present study suggested that anti-apoptotic proteins may serve as potential therapeutic targets for the protection of damaged brain in cases of kainic acid-seizure induction. Previous studies have shown that the administration of Ganoderma lucidum extracts (containing polysaccharides and ergosterol) exert neuroprotective effects by the production of microglia-derived proinflammatory and cytotoxic factors. The results obtained by us show for first time that G. curtisii mycelium produces polysaccharide with neuroprotective activity on rats subjected to kainic acid-seizure induction, and that an apoptotic mechanism may be involved in the neuroprotective effect of GCPS-2.

Neurotoxicity events in the brain following epileptic seizures implies increase in apoptotic signaling molecules and in the levels of inflammatory mediators (IL- $1 \beta$, TNF- $\alpha$, etc). The control of these 


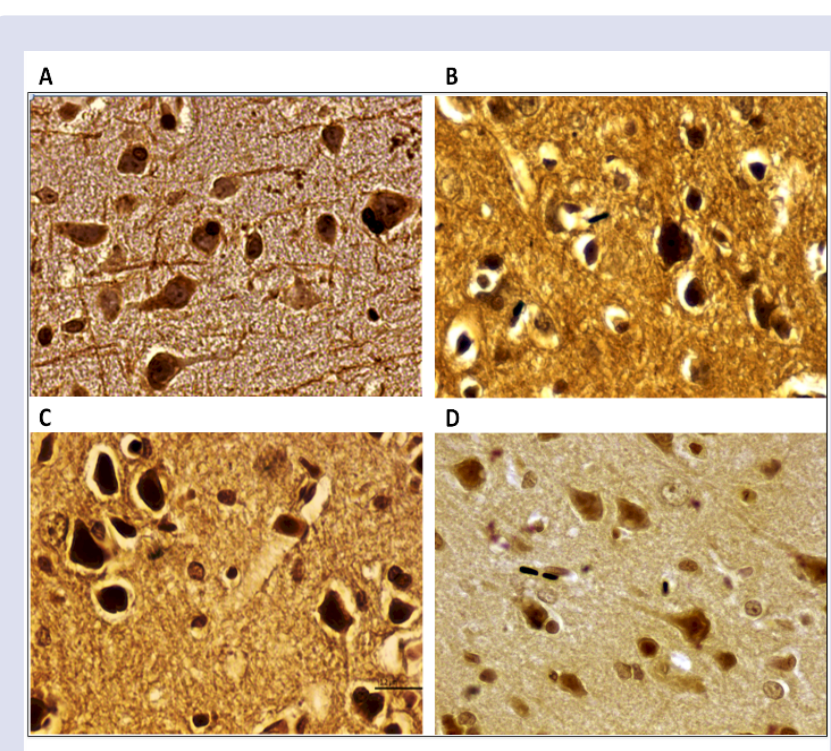

E

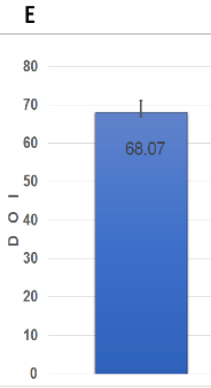

Control

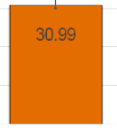

GCPS-2

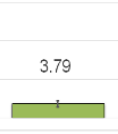

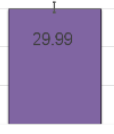

GCPS-2+KA
Figure 7: Photomicrographs of cortex tissue sections: A) control group showing normal neurons and NeuN level; B) GCPS-2 group showing interstitial edema; C) kainic acid group showing abundant neuronal loss and neurodegeneration; D) GCPS-2 + KA group showing less degenerate neurons and normal NeuN expression similar to control group; E) A smaller number of cells were observed in the animals treated with KA, the group treated with GCPS-2 + KA, showed an expression similar to that of the control group.

inflammatory pathways may be one the many potential strategies for the prevention of seizures. The results of this study demonstrate that pretreatment with GCPS-2 inhibit microglial activation in KA-treated hippocampus and cortex of rats, resulting in a decrease of levels of IL$1 \beta$ and TNF- $\alpha$. These results suggest that the anti-apoptotic and antiinflammatory effects of GCPS-2 are associated with the protection of hippocampal and cortex neurons from KA-induced neurotoxicity.

The present study showed the neuroprotective effects of GCPS-2 isolated from a Ganoderma curtisii strain collected in Mexico, against KAinduced seizures in vivo. Our results demonstrate that GCPS-2 inhibit convulsions in KA-seizure induced model. In addition, we determined that the neuroprotective properties of GCPS-2 against KA-induced excitotoxicity is mediated by the inhibition of microglial activation and apoptotic signaling molecules in hippocampus and cortex. These results suggest that GCPS-2 is a potential natural compound for the prevention and treatment of epileptic seizure.

\section{ACKNOWLEDGMENTS}

We thank B. Eda (CIQ, UAEM) for the recording of NMR spectra. V. Núñez and J. Méndez are grateful to CONACYT for scholarships (572999 and 21633). We also thank to Gabriela León (Fac. Química, UNAM) for technical assistance. We are very grateful to LANEM for allowing access to Analytical Equipment.

\section{REFERENCES}

1. World health organization (WHO). Atlas: epilepsy care in the world WHO Geneva, 2005.

2. Lévesquea M, Avolia M, Bernardd C. Animal models of temporal lobe epilepsy following systemic chemoconvulsant administration. J Neurosci Methods. 2016;260:45-52

3. Perucca E. An introduction to antiepileptic drugs. Epilepsia. 2005;46:31-7.

4. Zhoua Z, Yu-Ping T, Jun X, Pin W, Hui-Ming J, Zhong W, et al. Neuroprotective effects of water-soluble Ganoderma lucidum polysaccharides on rat. J Ethnopharm. 2010;131:154-64.

5. Banothu V, Neelagiri C, Adepally U, Lingam J, Bommareddy K. Phytochemical screening and evaluation of in vitro antioxidant and antimicrobial activities of the indigenous medicinal plant Albizia odoratissima. Pharm Biol. 2017;55:115561.

6. Kuan-Ming C, Tzu-Yu L, Ming-Lee Y, Cheng-Wei L, Ming-Jiuh W, Su-Jane W. Dexmedetomidine protects neurons from kainic acid-induced excitotoxicity by activating BDNF signaling. Neurochem Int. 2019;109:104493.

7. Ruiping Z, Shengli X, Yanning C, Ming Z, Xiaohong Z, Piu C. Ganoderma lucidum protects dopaminergic neuron degeneration through inhibition of microglial activation. Evid Based Complement Altern Med. 2009;2011:1-9.

8. Zhang P, Cheung P. Evaluation of sulfated Lentinus edodes alpha-(1-3)-D-glucan as a potential antitumor agent. Bioscience Biotech Biochem. 2002;66:1052-6.

9. Bao X, Liu C, Fang J, Li X. Structural and immunological studies of a major polysaccharide from spores of Ganoderma lucidum (Fr.) Karst. Carbohyd Res. 2001;332:67-74

10. Aguilar I, Molina J, Garnica MG, Yahuaca B. Total polyphenols and antioxidant activity of Ganoderma curtisii extracts. J Med Plants Studies. 2016;4:136-41.

11. Yang J, Ting X, Lu-Hui Z, Qian W, Li Q, Li-Xia C. Lanostane triterpenoids from Ganoderma curtisii and their NO production inhibitory activities of LPS-induced microglia. Bioorg Med Chem. 2016;26:3556-61.

12. Habijanic J, Berovic M, Boh B, Plankl M, Wraber B. Submerged cultivation of Ganoderma lucidum and the effects of its polysaccharides on the production of human cytokines TNF-a, IL-12, IFN-g, IL-2, IL-4, IL-10, and IL-17. New Biotech. 2015;32:85-95

13. Russell R, Paterson M. Ganoderma, a therapeutic fungal biofactory Phytochemistry. 2006;67:1985-2001.

14. Socala K, Nieoczym D, Grzywnowicz K, Stefaniuk D, Wlaz P. Evaluation of anticonvulsant, antidepressant-, and anxiolytic-like effects of an aqueous extract from cultured mycelia of the Lingzhi or Reishi medicinal mushroom Ganoderma lucidum (Higher Basidiomycetes) in mice. Int J Med Mushrooms. 2015;17:209-18.

15. Vyas D, Seikh IA, Tiwari GK. Role of mushroom in maintaining mental health with special reference to anti-convulsant activity. The Int J Indian Psychology. 2016;4:73-92.

16. Aguirre-Moreno A, Villeda-Hernández J, Campos-Peña V, Herrera-Ruiz M, Montiel E, Tello I, et al. Anticonvulsant and neuroprotective effects of oligosaccharides from Lingzhi or Reishi medicinal mushroom, Ganoderma lucidum (Higher Basidiomycetes). Int J Med Mushrooms. 2013;15:555-68.

17. Tokuhara D, Sakuma S, Hattori H, Matsuoka Q, Yamano T. Kainic acid dose effects delayed cell death mechanism after status epilepticus. Brain Dev. 2007;48(Suppl 2):13-20.

18. Man-He Z, Xiu-Min Z, Jian-Zhong C, Kai-Jie W, Yan F, Hong-Ao Z. Neuroprotective effects of dexmedetomidine on traumatic brain injury: Involvement of neuronal apoptosis and HSP70 expression. Mol Med Rep. 2018;17:8079-86. 


\section{GRAPHICAL ABSTRACT}
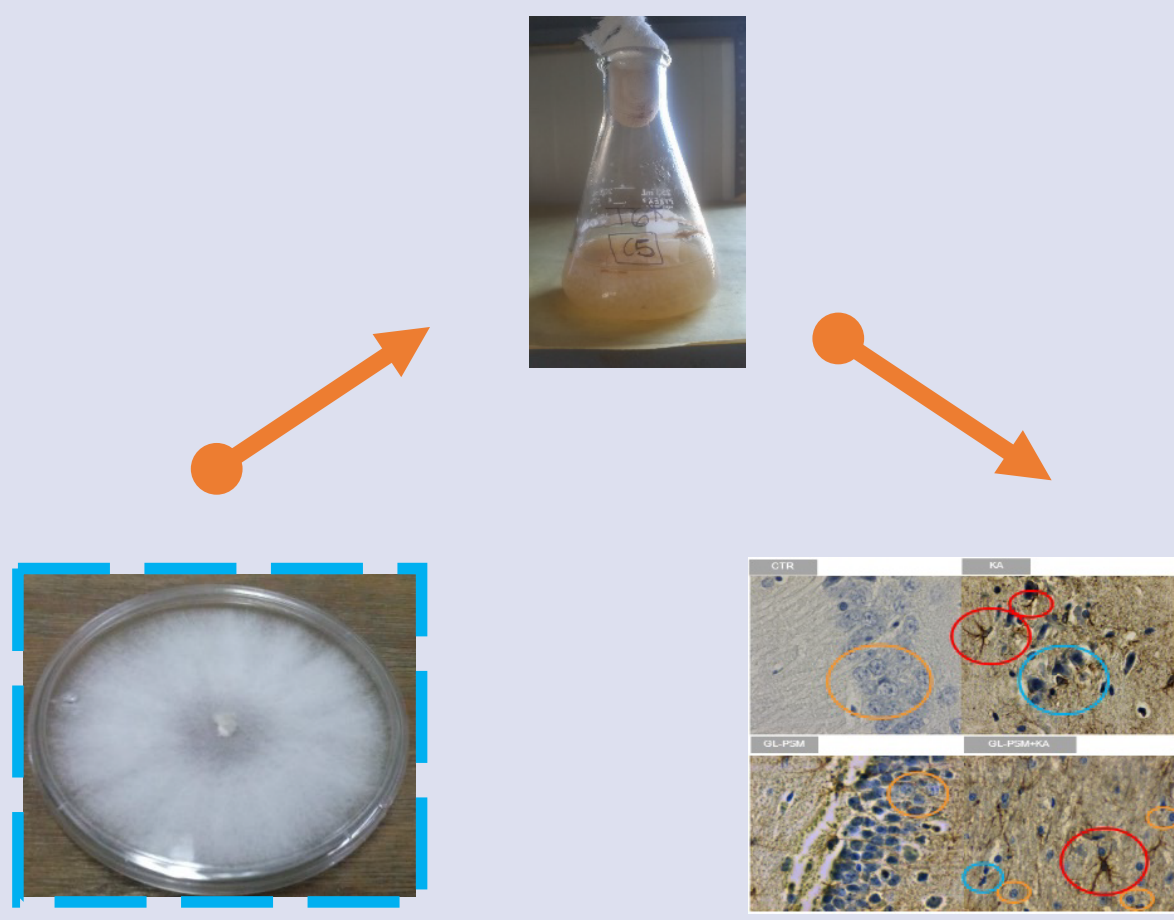

\section{ABOUT AUTHORS}

Dr. Ismael León Rivera. Earned his Bachelor in Chemistry, M. Sc. And PhD in Chemical Sciences at Universidad Nacional Autónoma de México. His M. Sc, and PhD research were focused on the isolation and characterization of resin glycosides from Ipomoea stans by NMR methodologies. Currently, Dr. León is a Lecturer and Researcher at Centro de Investigaciones Químicas, Universidad Autónoma del Estado de Morelos. His research interests are the isolation, characterization, and evaluation of the neuroprotective activity of saccharides from medicinal plants (Ipomoea species) and mushrooms (Ganoderma species).

Dra. Juana Villeda Hernández (1957 - 2018) studied her Bachelor and M. Sc. Degree at Facultad de Ciencias, Universidad Nacional Autónoma de México. She got the PhD degree at Universidad Autónoma Metropolitana (Unidad Iztapalapa) performing studies with exotoxicity on rat brain. She was a Researcher at Instituto Nacional de Neurología y Neurocirugía, developing the study of neurodegenerative diseases. This manuscript is dedicated to her Memory.

M. Sc. Elizur Montiel Arcos studied his Bachelor at Faculty of Biological Sciences, Universidad Autónoma del Estado de Morelos (UAEM). Since his experimental work of Thesis he has been interested in studying mushrooms. He got the degree of Master in Sciences at Universidad Nacional Autónoma de México, also with mushrooms as theme. He is currently the Responsible of Mycology Laboratory, Centro de Investigaciones Biológicas, UAEM. His research is focused in the study of medicinal mushrooms.

Dr. Isaac Tello Salgado studied his Bachelor in Faculty of Biological Sciences, at Universidad Autónoma del Estado de Morelos (UAEM). He got the PhD degree at Colegio de Posgraduados, Puebla, México, performing the molecular study of several strains of mushrooms collected in Mexico as his theme of Thesis. He is currently a Researcher in the Mycology Laboratory, Centro de Investigaciones Biológicas, UAEM. His research is focused in the study of medicinal mushrooms.

Dra. María Yolanda Rios completed her PhD at Universidad Nacional Autónoma de México in 1996. She was appointed at Universidad Autónoma del Estado de Morelos, as a Professor within the Natural Products Chemistry Department from 1992 to the present day. She was a visiting professor in 2001, 2007 and 2012 at the University of lowa, USA. Furthermore, Yolanda has authored close to one hundred scientific publications in international journals and has supervised the research work of a large number of PhD, Master and Bachelor students as well as seven Postdoctoral Fellows. To learn more about her work visit http:// yolanda-rios.net.

Dr. Samuel Estrada Soto. His Bachelor, Master and PhD degrees were obtained at National Autonomous University of Mexico. Currently, he is the leader of the Pharmacognosy Research Group in Pharmacy Faculty at Autonomous University of Morelos State, Mexico. He has presently published 112 scientific articles in indexed and international circulation journals. His main line of research is directly related to the search for drugs from natural and semi-synthetic products with pharmacological potential in the treatment of asthma, diabetes, hypertension and obesity. 
Dra. Angélica Berenice Aguilar Guadarrama is a Researcher at Universidad Autónoma del Estado de Morelos. She is interested in the phytochemical study of Mexican plants, some of them used in Mexican Traditional Medicine. The main goals of her investigations are determination of chemical structure of secondary metabolites and the establishment of the biological activity of extracts and compounds on in vitro assays.

M. Sc. Verónica Núñez Urquiza studied her Bachelor in Faculty of Chemical Sciences and Engineer, at Universidad Autónoma del Estado de Morelos (UAEM). She got the M. Sc. degree at Centro de Investigaciones Químicas (CIQ, UAEM) performing the production and characterization of exopolysaccharide by submerged culture of Ganoderma lucidum mycelium. She is currently developing her PhD Thesis at (CIQ, UAEM) on the biotechnological production of bioactive compounds from a new Ganoderma species supervised by Dr. Ismael León.

Dra. Victoria Campos Peña studied her Bachelor in Experimental Biology, at Universidad Autónoma Metropolitana (Unidad Iztapalapa). She got the M. Sc. (Molecular Biomedicine) and PhD degrees at Centro de Investigación y Estudios Avanzados performing studies with the neuropathology of Alzheimer. She did a Research stay at Northwestern University Medical School in 2000. Currently she is a Researcher at Instituto Nacional de Neurología y Neurocirugía, developing the study of neurodegenerative diseases.

Jasmín Méndez Mirón studied his Bachelor in Faculty of Biological Sciences, at Universidad Autónoma del Estado de Morelos (UAEM). Part of the experimental work of her BachelorThesis was performed in Laboratorio de Enfermedades Neurodegenerativas, at Instituto Nacional de Neurología y Neurocirugía.

Dr. Sergio Hidalgo Figueroa studied in Faculty of Pharmacy at Autonomous University of Morelos State (B.Sc., M.Sc. and Ph.D.) as part of his training as an Organic Medicinal Chemistry. He performed a Postdoctoral stay in the Autonomous Metropolitan University. Currently, he is Professor of Chemistry at Potosi Institute of Scientific and Technological Research. His research focus in the development of: multifunctional or multitarget compounds, pharmacological experiments (experimental models of diabetes and hypertension), bioinformatics and computational chemistry. His research projects are multidisciplinary, addressing problems at chronic degenerative diseases.

M. Sc. Eva Hernández studied her Bachelor in Faculty of Biological Sciences, at Universidad Autónoma del Estado de Morelos (UAEM). She got the degree of Master in Sciences at Universidad Autónoma del Estado de Morelos, working with medicinal plants. She has been working for Instituto Nacional de Salud Pública for many years. Her research is focused in the study of medicinal plants.

M. Sc. Gerardo Hurtado studied his Bachelor in Faculty of Chemical Sciences and Engineer, at Universidad Autónoma del Estado de Morelos (UAEM). He got the degree of Master in Sciences at Universidad Autónoma del Estado de Morelos. He has been working for Instituto Nacional de Salud Pública at the Proteomic Unit. His research is focused in the analytical characterization of bioactive metabolites.

Cite this article: León-Rivera I, Villeda-Hernández J, Montiel-Arcos E, Tello I, Rios MY, Estrada-Soto S, et al. Neuroprotective Effects of Ganoderma curtisii Polysaccharides After Kainic Acid-Seizure Induced. Pharmacog J. 2019;11(5):1046-54. 